\title{
Spatial autocorrelation analysis in plant population: An overview
}

\author{
Manish Mathur \\ 18E/564 Chopasni Housing Board, Jodhpur (Rajasthan), INDIA \\ Present Address: Plant Ecology Laboratory, Central Arid Zone Research Institute, Jodhpur (Rajasthan), INDIA \\ E-mail: eco5320@gmail.com \\ Received: September 4, 2014; Revised received: December 27, 2014; Accepted: January 20, 2015
}

\begin{abstract}
Analysis of spatial distribution in ecology is often influenced by spatial autocorrelation. In present paper various techniques related with quantification of spatial autocorrelation were categorized. Three broad categories namely global, local and variogram were identified and mathematically explained. Local measurers captures the many local spatial variation and spatial dependency while global measurements provide only one set of values that represent the extent of spatial autocorrelation across the entire study area. Global spatial autocorrelation measures the overall clustering of data and it included six well defines methods, namely, Global index of spatial autocorrelation, Joint count statistics, Moran's I, Geary's C ration, General G-statistics and Getis and Ord's G. The study revealed that out of the six methods Moran's I index was most frequently utilized in plant population study. Based on their similarity degree, local indicator of spatial association (LISA) can differentiate the neighbors in to hot and cold spots. Correlogram and variogram approaches are also given.
\end{abstract}

Keywords: Correlogram, Global and Local Autocorrelation, Moran's I Spatial Autocorrelation, Variogram approaches

\section{INTRODUCTION}

When a plant population or community is sampled, the samples have a spatial relationship with each other. To a certain extent, samples that are close to each other are more likely to be similar (Dale, 1999). For example if vegetation is sampled using a transect of small contiguous quadrates, adjacent quadrats are likely to be more similar than those at greater spacing. The lack of independence is referred to as to as spatial autocorrelation because the correlation occurs within the data set itself and arises because of spatial relationships.

Spatial autocorrelation may be defined as the relationship among the values of a single variable that comes from the geographic arrangement of the areas in which these values occur. It measures the similarity of objects within an area; the degree to which a spatial phenomenon is correlated to itself in space (Cliff and Ord, 1981), the level of interdependence between the variables, the nature and strength of the interdependence, i.e. spatial autocorrelation is an assessment of the correlation of a variable in reference to spatial location of the variable. Assess if the values are interrelated, and if so is there a spatial pattern to the correlation, i.e. is there spatial autocorrelation. Weixelman and Riegel (2012) have utilized spatial autocorrelation to understand how to sample the community in mountain meadows and they have concluded that quantification of sampling point spacing is the prerequisite to ensure spatial and statistical independence for the presence/absence data. Roe et al., (2012) have questioned that how does the spatial structure of plant communities vary with the spatial grain and with the measure of species presence used and how can communities most efficiently be sample for spatial autocorrelation?. They have sampled the area with using triabgular sampling scheme. In their study the percentage variation in species composition explained by distance, i.e. by spatial autocorrelation, was higher at larger grain. However, it reached a maximum of only $15 \%$. The nugget - the Y-intercept of the dissimilarity/ distance relation - has been seen as a measure of randomness in community composition. It was generally about 0.5 dissimilarity on a $0-1$ scale, although values in the range $0.7-0.8$ were found at smaller grain sizes in the forest. The $90 \%$ distance, i.e. the distance at which dissimilarity reaches $90 \%$ of its final value, was interpretable only for the two sites where spatial autocorrelation was strong, but gave realistic estimates. On the basis of these results they have concluded that lack of increased spatial community predictability when including species abundances conforms to the majority of previous studies, indicated that the primary community control is on the presence of species, not their abundance. They have argued that the triangular sampling scheme used here was rapid, accurate, and efficient in its distribution of distances.

The causes of spatial autocorrelation are manifold, but three factors particularly common (Legendre and Fortin, 1989; Legendre, 1993; Legendre and Legendre, 1998) are:1) biological processes such as speciation, extinction, dispersal or species interactions are distance -related; 2) non-linear relationships between environment and species are modeled erroneously as linear; 3) the statistical model fails to account for an important environmental determinant that in itself is spatially 
structured and thus causes spatial structuring in the response (Besag, 1974). The second and third points are not always referred to as spatial autocorrelation, but rather spatial dependency (Legendre et al., 2002). Since they also lead to autocorrelated residuals, these are equally problematic. A fourth source of spatial autocorrelation relates to spatial resolution, because coarse grains lead to a spatial smoothing of data. In all of these cases, spatial autocorrelation may confound the analysis of species distribution data. Spatial autocorrelation may be seen as both an opportunity and a challenge for spatial analysis. It is an opportunity when it provides useful information for inference of process from pattern (Palma et al., 1999), for example, increasing our understanding of contagious biotic processes such as population growth, geographic dispersal, differential mortality, social organization or competition dynamics (Griffith and Peres-Neto, 2006). In most cases, however, the presence of spatial autocorrelation is seen as posing a serious shortcoming for hypothesis testing and prediction (Lennon, 2000; Dormann, 2007), because it violates the assumption of independently and identically distributed (i.e.) errors of most standard statistical procedures (Anselin, 2002) and hence inflates type I errors, occasionally even inverting the slope of relationships from non-spatial analysis (Ku“hn, 2007). Spatial autocorrelation describes the correlation of the values of a single attribute related to the proximity of those values in geographic space (Tobin, 2004; Suzuki et al., 2008). Spatial autocorrelation relies on data interdependence and spatial statistics (Odland, 1988 and Overmars et al., 2003).

Spatial autocorrelation exists when there is systematic spatial variation in the values of a given variable. This variation can exist in two forms called positive or negative spatial autocorrelation. In the positive case, the value of a variable at a given location tends to be similar to the values of that variable in nearby locations. This means that if the value of some variable is low in a given location, the presence of spatial autocorrelation indicates that nearby values are also low. Conversely, negative spatial autocorrelation is characterized by dissimilar variant values in nearby locations. For example, a low variant value may be surrounded by high values in nearby locations when negative spatial autocorrelation exists. A positive spatial autocorrelation refers to a map pattern where geographic features of similar value tend to cluster on a map, whereas a negative spatial autocorrelation indicates a map pattern in which geographic units of similar values scatter throughout the map. Positive spatial autocorrelation occurs when adjacent neighborhood locations have similar attribute values (Griffth, 2003; Almeida-Neto and Lewinsohn, 2004 Mueller-Warrant et al., 2008). When no statistically significant spatial autocorrelation exists, the pattern of spatial distribution is considered random (Chou, 1997). Positive spatial autocorrelation over short distances is commonly observed and reflects phenomena that are contagious (clumped) over short distance, such a limited seed dispersal, clonal growth, nutrient-rich patches and positions on an environmental gradient. By contrast, negative spatial autocorrelation over short distances would suggest avoidance or competition among neighbors.

Methods dealing with spatial autocorrelation: Spatial autocorrelation tools test whether the observed value of a variable at one locality is independent of values of the variable at neighboring localities. A variety of methods have consequently been developed to correct for the effects of spatial autocorrelation (partially reviewed by Miller et al., 2007), but only a few have made it into the ecological literature.

Before considering the use of modeling methods that account for spatial autocorrelation, it is a sensible first step to check whether spatial autocorrelation is in fact likely to impact the planned analyses, i.e. if model residuals indeed display spatial autocorrelation. Checking for spatial autocorrelation (SAC) has become a commonplace exercise in geography and ecology (Sokal and Oden, 1978 and Fortin and Dale, 2005). There are many indicators of spatial autocorrelation (Cliff and Ord, 1981; Goodchild, 1986; Isaaks and Shrivastava 1989; Haining, 1990; Chou, 1997; Perry et al., 2002; Su-Wei and Hsieh 2010). Diniz-Filho et al. (2003) and Kuhn (2007) have argued that the presence of residual spatial autocorrelation should always be tested for in spatial ecology and appropriate methods should be used if there is shown to be significant spatial autocorrelation

Spatial autocorrelation is a widespread phenomenon in ecology (Fortin et al., 1989; Henebry, 1995; Torgersen et al., 1995; Koenig, 1998; Radeloff et al., 2000). The spread of plants by the processes of growth, vegetative reproduction, and limited seed dispersal produces spatial autocorrelation. Plant species respond individualistically to their environment, both directly and indirectly through species interactions. Autocorrelation can be used to compare the pattern of the same species in different environments, and the patterns of different species in the same environment. For plant analysis the spatial autocorrelations and its various uses were discussed by Heikkinen et al. (1996), Hawkins et al. (2003), Dorman (2007) and Su-Wei and Hsieh (2010). Goslee (2006), Roe et al., (2012), Weixelman and Riegel (2012) and Mathur (2014) have discussed the behaviors of different vegetation sampling methods in the presence of spatial autocorrelation. He concluded that transect methods especially responded poorly to spatial autocorrelation and randomly-located quadrats were more efficient in highly patchy environments, but large numbers of quadrats are needed for either method if rare species must be located. He emphasized that multi-scale methods are more robust to spatial autocorrelation, and more effective at identifying rare species because of the larger proportion of the total area sampled. Cover and especially frequency estimates are highly variable, 
and depended on both the level of autocorrelation and the sampling method used.

Spatial autocorrelation can be analyzed from two different perspectives. Global autocorrelation analysis involves the study of the entire map pattern and generally asks the question as to whether the pattern displays clustering or not. Local autocorrelation, on the other hand, shifts the focus to explore within the global pattern to identify clusters or so called hot spots that may be either driving the overall clustering pattern, or that reflect heterogeneities that depart from global pattern. Global statistics answer the question: is there a spatial pattern $(\mathrm{Y} / \mathrm{N})$ ? Local statistics, on the other hand, answer the question: where is there spatial pattern (map)

1. Global indicators of spatial autocorrelation includes methods like Gamma index of spatial autocorrelation, Join count statistics, Moran's I, Geary's C, and Getis and Ord's G

2. Local indicators of spatial autocorrelation includes local Moran's I, Local Geary and Local Gi and Gi* statistics.

3. The variogram approach to spatial association (geostatistical perspective) includes correlogram and semi variogram

Defining contiguity:In spatial autocorrelation analysis some measure of contiguity is required. Contiguity has a rather broad definition depending on the research question, however, most analyses in spatial autocorrelation adhere to a common definition of neighbourhood relations. Namely, neighbourhood relations are defined as either rooks case, bishops case or queens (kings) case. These are rather simple and intuitive as their names suggest (Fig. 1). Rooks case contiguity is by a neighbourhood of 4 locations adjacent to each cell, Bishops only considers the diagonals of the relation and queens or kings case considers a neighbourhood of eight cells. These are the most common forms of contiguity used in spatial autocorrelation when considering continuous data in a raster format. Of these three the rooks case is the most commonly used and most programmes only will compute this case.

\section{Global autocorrelation}

Global spatial autocorrelation is a measure of the overall clustering of the data. Global indices of spatial autocorrelation have been used to evaluate the degree to which similar observations tend to occur near each other (Rogerson, 1999; Waller and Gotway, 2004 and Jackson and Waller, 2005). Six different tests for global spatial autocorrelation includes the Gamma index of spatial autocorrelation, join count statistics, Moran's I, Geary's C, General G statistics and Getis and Ord's G. The common elements of the various spatial autocorrelation models are (1) a matrix of values representing the association between locations and (2) values representing a vector of the attributes of the various locations (Getis, 1991).

A. Gamma index of spatial autocorrelation: The Gamma Index of spatial autocorrelation consists of the application of the principle behind a general crossproduct statistic to measuring spatial autocorrelation Hubert et al. (1981). The idea is to assess whether two similarity matrices for $n$ objects, i.e., $n$ by $n$ matrices $A$ and B measure the same type of similarity. This is reflected in a so-called Gamma Index $\Gamma=\sum_{i} \sum_{j} a_{i j} \cdot b_{i j}$

In other words, the statistic consists of the sum over all cross-products of matching elements $(i, j)$ in the two matrices. The application of this principle to spatial autocorrelation consists of turning the first similarity matrix into a measure of attribute similarity and the second matrix into a measure of locational similarity. Naturally, the second matrix is the spatial weight matrix. The first matrix can be any reasonable measure of attribute similarity or dissimilarity, such as a cross-product, squared difference or absolute difference.

Formally, then, the Gamma index is:

$$
\boldsymbol{\Gamma}=\sum_{i} \sum_{j} a_{i j} \cdot w_{i j}
$$

Where $w_{i j}$ are the elements of the weights matrix and $a_{i j}$ are corresponding measures of attribute similarity. Inference for this statistic is based on a permutation approach in which the values are shuffled around among the locations and the statistic is recomputed each time. This creates a reference distribution for the statistic under the null hypothesis of spatial randomness. The observed statistic is then compared to this reference distribution and a pseudo-significance computed as $p=(m+1) /(n+1)$

Where $m$ is the number of values from the reference
Rooks moves

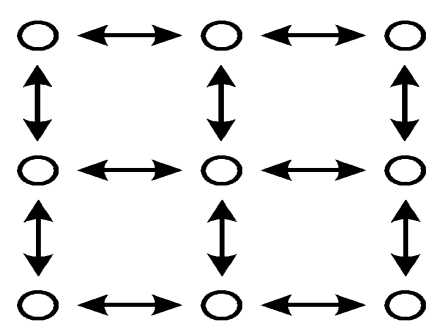

Bishop's moves

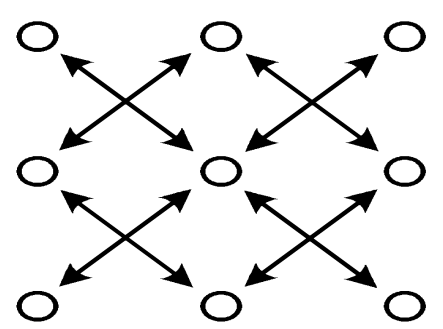

\section{Queen's moves}

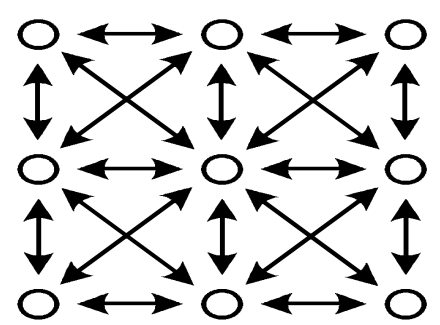

Fig. 1. Type of neighbourhoods relations. (Mathur 2014). 
distribution that are equal to or greater than the observed join count and $n$ is the number of permutations. The Gamma test is a two-sided test in the sense that both extremely high values (e.g., larger than any value in the reference distribution) and extremely low values (e.g., smaller than any value in the reference distribution) can be considered to be significant. Depending on how the measure of attribute similarity is defined, a high value will indicate positive or negative spatial autocorrelation, and vice versa. For example, for a cross-product measure of attribute similarity, high values indicate positive spatial autocorrelation and low values negative spatial autocorrelation. For a squared difference measure, it is the reverse. This is similar to the interpretation of the Moran's I statistic and Geary's $C$ statistic respectively. Many spatial autocorrelation test statistics can be shown to be special cases of the Gamma index. In most instances, the Gamma index is an unstandardized version of the commonly used statistics. As such, the Gamma index is scale dependent, since no normalization is carried out (such as deviations from the mean or rescaling by the variance).

B. Joint count statistics: Join-count statistics are the simplest measure of spatial autocorrelation which were put forward by Moran in 1948. They are used for a binary variable, "0" and "1", which are often used referred to as "white" and "black". A join links two neighboring areas; the possible types of joins are limited to white-white (WW), black-white (BW), and black-black (BB). Joincounts are frequencies or counts of the numbers of $(0,0)$, $(0,1)$, and $(1,1)$ joins in the study area, and these numbers are compared to the expected numbers of them under the null hypothesis of no spatial autocorrelation. The observed number of $(0,0),(0,1)$ and $(1,1)$ joins are given by

$$
\begin{aligned}
& (0,1)=\frac{\sum_{i=1}^{n} \sum_{j=1}^{n} C_{i j}\left|x_{i}-x_{j}\right|}{2} \\
& (0,0)=\frac{\sum_{i=1}^{n} \sum_{j=1}^{n} C_{i j}\left(1-x_{i}\right)\left(1-x_{j}\right)}{2} \\
& (1,1)=\frac{\left.\sum_{i=1}^{n} \sum_{j=1}^{n} C_{i j} x_{i} x_{j}\right)}{2}
\end{aligned}
$$

Where $x i$ is the binary attribute value related to region $\mathrm{i}, 1$ or $0, C i j$ is a value assigned to region $\mathrm{i}$ and region $\mathrm{j}$ by a spatial weighting function. Join-count statistics have been widely applied to analysis spatial autocorrelation in remote sensing data (Congalton, 1988; Chuang and Huang, 1992; Pugh and Congalton, 2001). Most of these applications on join-count are restrained on binary variables, the values of each spatial unit are either 1 (black) or 0 (white).

C. Moran's I: Moran (1948) introduced the first measure of spatial autocorrelation in order to study stochastic phenomena which are distributed in space in two or more dimensions. Moran's I has been subsequently used in almost all studies employing spatial autocorre- lation (Upton and Fingleton, 1985). Measuring the correlation (simultaneous change in value of two numerically valued random variables) among the neighboring observations in the pattern is done using the spatial autocorrelation statistic Moran's I. Moran's I is defined as a measure of the correlation among neighboring observations in a pattern (Boots and Getis, 1988). Computation of Moran's $I$ is achieved by division of the spatial covariation by the total variation. Resultant values are in the range from approximately -1 to 1 . Positive signage represents positive spatial autocorrelation, while the converse is true for negative signage. With a Zero result representing no spatial autocorrelation.

$$
I=\frac{N \sum_{\mathrm{i}=1}^{\mathrm{n}} \sum_{\mathrm{j}=1}^{\mathrm{n}} \mathrm{w}_{\mathrm{ij}}\left(\mathrm{x}_{\mathrm{i}}-\overline{\mathrm{x}}\right)\left(\mathrm{x}_{\mathrm{j}}-\overline{\mathrm{x}}\right)}{\left(\sum_{\mathrm{i}=1}^{\mathrm{n}} \sum_{\mathrm{j}=1}^{\mathrm{n}} \mathrm{w}_{\mathrm{ij}}\right) \sum_{\mathrm{i}=1}^{\mathrm{n}}\left(\mathrm{x}_{\mathrm{i}}-\overline{\mathrm{x}}\right)^{2}}
$$

Where $N$

is the number of observation of the whole region

$\mathrm{X}$ is the mean of the variable

$\mathrm{X}_{\mathrm{i}}$ is the variable value at a particular location

$\mathrm{X}_{\mathrm{j}}$ is the variable value at another location

$\mathrm{W}_{\mathrm{ij}}$ is a spatial weight between locations of $i$ relative to $j$

Moran's I formula is related to Pearson's correlation coefficient; its numerator is a covariance, comparing the values found at all pairs of points in turn, while its denominator is the maximum-likelihood estimator of the variance (i.e. division by $n$ instead of $n-1$ ); in Pearson's $r$, the denominator is the product of the standard deviations of the two variables, whereas in Moran's I there is only one variable involved. Moran's I mainly differs from Pearson's $r$ in that the sums in the numerator and denominator do not involve the same number of terms; only the terms corresponding to distances within the given class are considered in the numerator whereas all pairs are taken into account in the denominator.

Statistical test for Moran's I: Global Moran's I can be standardized to $\mathrm{Z}$ and this can be calculated as

$$
Z=\frac{\mathrm{I}-\mathrm{E}(\mathrm{I})}{\sqrt{\mathrm{V( \textrm {I } )}}}
$$

$\mathrm{E}(\mathrm{I})=-1 /(\mathrm{n}-1)$

Where, $W_{-j *}$ is the sum of all weights located in the row $\mathrm{I}, \mathrm{W} *_{\mathrm{i}}$ is the sum of all weights in the column $\mathrm{i}$. The threshold of 1.96 can be applied to test the significance level of $\mathrm{Z}$. If $\mathrm{Z}$ is greater than 1.96 or smaller than

$$
\begin{aligned}
& \operatorname{Var}(l)-\frac{1}{w_{n}^{2}\left(n^{2}-1\right)}\left(n^{2} w_{1}-n w_{2}+3 w_{0}^{2}\right)-Z^{2}-(l) \\
& w_{0}=\sum_{i=1}^{n} \sum_{j=1}^{n} w_{i j} ; w_{1}=0.5 \sum_{i=1}^{n} \sum_{j=1}^{n}\left(w_{i j}+w_{j i}\right)^{2} ; w_{2}=\sum_{i=1}^{n}\left(w_{i}+w_{i j}\right)^{2}
\end{aligned}
$$

-1.96, it implies that the spatial autocorrelation was significant (Zhang and McGrath, 2004) 
The tool computes a z-score and p-value indicating whether this difference is statistically significant or not. Index values cannot be interpreted directly; they can only be interpreted within the context of the null hypothesis. The p-value is a probability. For the pattern analysis tools, it is the probability that the observed spatial pattern was created by some random process. When the p-value is very small, it means it is very unlikely (small probability) that the observed spatial pattern is the result of random processes, so we can reject the null hypothesis. Z-scores are simply standard deviations. Both z-scores and p-values are associated with the standard normal distribution (Table 1)

Very high or very low (negative) z-scores, associated with very small $\mathrm{p}$-values, are found in the tails of the normal distribution. When we run a feature pattern analysis tool and it yields small p-values and either a very high or a very low $\mathrm{z}$-score, this indicates it is unlikely that the observed spatial pattern reflects the theoretical random pattern represented by our null hypothesis (CSR). A statistically significant positive $\mathrm{z}$ score means that similar values cluster spatially. High values are found closer together, and low values are found closer together, than we would expect from an underlying random spatial process. A statistically significant negative $\mathrm{Z}$ score means that similar values are spatially dispersed. High values are found far away from other high values, and low values are found far away from other low values, and this dispersion is more pronounced than we would expect from an underlying random spatial process (Table 2).

The spatial autocorrelation (Global Moran's I) tool is an inferential statistic, which means that the results of the analysis are always interpreted within the context of its null hypothesis. For the Global Moran's I statistic, the null hypothesis states that the attribute being analyzed is randomly distributed among the features in study area.

Dray et al. (2008) have introduced multivariate spatial analysis based on Moran;s I (MULTISPATI) by introducing a row-sum standardized spatial weight matrix. Their analysis implies a compromise between relations among variables (multivariate analysis) and their spatial structure (autocorrelation). Chen et al. (2008) have quantified the strength of autocorrelation by using the Moran's I. They have reported that for both $\alpha$ and $\beta$ tree diversities, the distance of the spatial autocorrelation increased slightly at the scale of 10-15 and $200 \mathrm{~km}$ and decreased around 20-40 km.

Modified version of Moran's I: Traditional calculation of Moran's I for heterogeneous populations is not working well (Jackson et al., 2010). Therefore, several alternative version of Moran's I have been proposed to account for heterogeneous population, for example Oden (1995); Waldhor (1996); Assuncao and Reis (1999) and Waller et al. (2006). Oden (1995) proposed the modified Moran's I function (I*pop) he noted that symmetry is not required for $\mathrm{I}^{*}{ }_{\text {pop }}$ and $\mathrm{w}_{\mathrm{ij} \#} 0$ (but can be fix at any specified value). In order to capture the variability present in the region, Oden includes the first term in the numerator which is used to model the spatial variation in a manner similar to conventional chisquared for heterogeneity rates. The odens's I* pop can be written as

Jackson et al. (2010) improves the original version of Moran's I by incorporation of (a) a weight function in the variance computation (b) introducing the population density weight function and (c) conducting Monte Carlo simulation. Their weight function is not only included in the differences of the geographic unit's

$$
\begin{aligned}
& I_{\text {pop }}^{*}=\left[n^{2} \sum_{i} \sum_{i} w_{i j}^{\left(\sigma_{i j}\right.}\left(\sigma_{i}-v_{i}\right)\left(\sigma_{i}-v_{i}\right)-n(1\right. \\
& \left.\left.-2 b^{b}\right) \sum_{i} w_{i j}^{*} \theta_{i}-n b^{\prime} \sum_{i} w_{i j}^{*} v_{i}\right] x \frac{1}{b^{\prime}\left(1-b_{i}\right)\left[n^{2}+\Sigma_{i} \Sigma_{j} v_{i} v_{j} w_{i j}^{*}-x \Sigma_{i} w_{i i j}^{*}\right]}
\end{aligned}
$$

cases from the overall mean, but also in the calculation of the variance. They also expand the definition of neighbors to a broader concept in the construction of Moran's I (e.g., all geographic units included in a pre-specified geographic range will be considered to be neighbors of the geographic unit in the center. They have concluded that modified Moran's I achieved higher power than Moran's I and I ${ }^{*}$ pop for evaluating global and local clustering patterns on geographic data with homogenous population D. Geary's $C$ (Contiguity) ratio: Computation of Geary's C results in a value within the range of 0 to +2 . With zero being a strong positive spatial autocorrelation, through to 2 , which represents a strong negative spatial autocorrelation? Calculation is similar to Moran's I, For Moran, the cross-product is based on the deviations from the mean for the two location values while for Geary, and the cross-product uses the actual values themselves at each location. Geary's C statistic (Geary, 1954) is based on the deviations in responses of each observation with one another:

$$
C=\frac{n-1}{2 S_{0}} \frac{\sum_{i} \sum_{j} w_{i j}\left(x_{i}-x_{j}\right)^{2}}{\sum_{i}\left(x_{i}-\bar{x}\right)^{2}} .
$$

The variance is:

$$
\begin{aligned}
\operatorname{Var}(c)= & \frac{1}{n(n-2)(n-3) S_{0}^{2}} \\
& \left\{S_{0}^{2}\left[\left(n^{2}-3\right)-k(n-1)^{2}\right]+S_{1}(n-1)\left[n^{2}-3 n+3-k(n-1)\right]\right. \\
& \left.+\frac{1}{4} S_{2}(n-1)\left[k\left(n^{2}-n+2\right)-\left(n^{2}+3 n-6\right)\right]\right\}
\end{aligned}
$$

Where SO, S1 and S2 are the same as in Moran's $I$. However, interpretation of these values is very different, essentially the opposite. Geary's $C$ varies on a scale from 0 to 2, $C$ of approximately 1 indicates no autocorrelation /random, $C$ of 0 indicates perfect positive autocorrelation /clustered, $\mathrm{C}$ of 2 indicates perfect negative autocorrelation /dispersed. Can convert to a $-/+1$ scale by: calculating $\mathrm{C}^{*}=1-\mathrm{C}$

Geary's $C$ coefficient is a distance-type function; it varies from 0 to some unspecified value larger than 1 . Its numerator sums the squared differences between 
values found at the various pairs of sites being compared. A Geary's $C$ correlogram varies as the reverse of a Moran's I correlogram; strong autocorrelation produces high values of $I$ and low values of $C$. Positive autocorrelation translates in values of $c$ between 0 and 1 whereas negative autocorrelation produces values larger than 1 . Hence, the reference 'no correlation' value is $C=1$ in Geary's correlograms.

Statistical significance tests for Geary's C: Similar to Moran which based on the normal frequency distribution with

$$
\begin{aligned}
& Z=\frac{C-E(C)}{S_{\text {errar }}(C)} \\
& \mathrm{E}(\mathrm{C})=1
\end{aligned}
$$

A statistically significant positive Z score means high/ larger values cluster spatially larger values are found closer together than we would expect if the underlying spatial process was random. A statistically significant negative $\mathrm{Z}$ score means that low/smaller values cluster spatially smaller values are found closer together than we would expect if the underlying spatial process was random.

Moran's I and Geary's $C$ coefficients are used to measure the degree of spatial autocorrelation displayed by a quantitative variable and to test the null hypothesis (Ho) that there is no significant spatial autocorrelation (positive: aggregation, segregation). Since these coefficients compare values for pairs of points, the set of available point pairs is divided into a number of distance classes. This number of classes is left to the user. Like Pearson's correlation coefficient, Moran's $I$ is based on the computation of cross products of centred data. Geary's $C$ is a distance type coefficient, summing squared differences between adjacent pairs of values. Spatial autocorrelation analysis should not be performed with fewer than 30 localities, because the number of pairs of localities in each distance class would then become too small to produce significant results (Cliff and Ord 1981; Legendre and Fortin 1989). Formulas for computing the coefficients as well as the standard error of the estimated statistics can be found in Cliff and Ord (1981), in Sokal and Oden (1978) and in Legendre and Legendre (1984)

Both Moran's I and Geary's $C$ are calculated at various distances, d. Both test the null hypothesis that there is no significance spatial autocorrelation among the mapped points with respect to the measured variable (Gibson, 2002 and Dormann et al., 2007). Moran's I is produced by standardizing the spatial auto covariance by the variance of the data using a measure of the connectivity of the data. Geary's $C$ uses the sum of squared differences between pairs of data values as its measure of covariation. Moran's I is a more global measurement and sensitive to extreme values of $\chi$, whereas Geary's $C$ is more sensitive to differences in small neighborhoods. In general, Moran's I and Geary's $C$ result in similar conclusions. However, Moran's I is preferred because it's more powerful than Geary's $C$. (Cliff and Ord, 1981)

\section{E. General G-Statistic}

Moran's I and Geary's $C$ will indicate clustering or positive spatial autocorrelation if high values (e.g. tree with high density) cluster together (often called hot spots) and/or if low values cluster together (tree with low density), but they cannot distinguish between these situations. The General $G$ statistic distinguishes between hot spots and cold spots. It identifies spatial concentrations. $G$ is relatively large if high values cluster together while $\mathrm{G}$ is relatively low if low values cluster together. The General $G$ statistic is interpreted relative to its expected value (value for which there is no spatial association). Larger than expected value indicates potential "hot spot" while smaller than expected value indicates potential "cold spot". Z test statistic is used to test if the difference is sufficient to be statistically significant.

Calculation of $G$ must begin by identifying a neighborhood distance within which cluster is expected to occur.

$$
G(d)=\frac{\Sigma \sum W_{i j}(d) x_{i} x_{j}}{\sum \sum x_{i} x_{j}}
$$

Where ; $d$ is neighborhood distance; $\mathrm{W}_{\mathrm{ij}}$ weights matrix has only 1 or $0 ; 1$ if $j$ is within d distance of $i$; 0 if its beyond that distance

\section{Statistical Significance Tests:}

$$
Z_{G}=\frac{E(G)}{\sqrt{V(G)}}
$$

Expected value (if no concentration) for $\mathrm{G}$ is given by: $\mathrm{V}_{\mathrm{G}}=\left(\mathrm{E}\left(\mathrm{G}^{2}\right)-\mathrm{E}(\mathrm{G})^{2}\right.$

$$
E(G)=\frac{W}{\mathrm{n}(\mathrm{n}-1)} \text { where } \mathrm{w}=\sum_{\mathrm{i}} \sum_{\mathrm{j}} w_{\mathrm{ij}}
$$

For the General G, the terms in the numerator (top) are calculated "within a distance bound (d)," and are then expressed relative to totals for the entire region under study. As with all of these measures, if adjacent $\mathbf{x}$ terms are both large with the same sign (indicating positive spatial association), the numerator (top) will be large

F. Getis and Ord's G:Method developed by Getis and Ord $(1992 ; 1996)$ not only provide hypothesis testing to determine whether clustering has occurred within a dataset, but also provide information on the extent to which above and below average values cluster more strongly and identify local concentration of clustering (Laffan, 2006; Mueller-Warrant et al., 2008). The high/low clustering (Getis-Ord General G) tool is an inferential statistic, which means that the results of the analysis are interpreted within the context of the null hypothesis. The null hypothesis for the high/low clustering (General G) statistic states that there is no spatial clustering of feature values. When the p-value returned by this tool is small and statistically significant, the null hypothesis 
can be rejected. If the null hypothesis is rejected, then the sign of the z-score becomes important. The high/low clustering (Getis-Ord General G) tool is most appropriate when we have a fairly even distribution of values and are looking for unexpected spatial spikes of high values. Unfortunately, when both the high and low values cluster, they tend to cancel each other out. The null hypothesis for both the high/low clustering (Getis-Ord General G) and the spatial autocorrelation (Global Moran's I) tool is complete spatial randomness (CSR); values are randomly distributed among the features in the dataset, reflecting random spatial processes at work. However, the interpretation of $\mathrm{z}$-scores for the high/low clustering tool is very different from the interpretation of z-scores for the spatial autocorrelation (Global Moran's I) tool: It measures the degree of clustering for either high values or low values. Where $y_{i}$ represent the value of feature $i, y_{j}$ represent the vlaue of feature $j$, and $\mathrm{w}_{\mathrm{ij}}$ is the weight assigned to each pair of features. Fox et al. (2012) have use global and local Moran's I spatial autocorrelation statistics, Getis-Ord General G global high/low clustering and Getis-Ord ${ }^{*}$ test statistic techniques to evaluate the spread patterns of pine beetle infestation over time.

Local Autocorrelation:Spatial autocorrelation can also be measured at the local level to evaluate the extent of autocorrelation within local neighbourhoods. Local measures captures the many local spatial variation and spatial dependency while global measurements provide only one set of values that represent the extent of spatial autocorrelation across the entire study area (Mueller -Warrant et al., 2008). As an operational definition, Anselin (1995) has defined the local indicator of spatial association (LISA) is as any statistic that satisfies the following two requirements: 1. the LISA for each observation gives an indication of the extent of significant spatial clustering of similar values around that observation; 2. the sum of LISAs for all observations is proportional to a global indicator of spatial association. The general LISA can be used as the basis for a test on the null hypothesis of no local spatial association. For each location, LISA values allow for the computation of its similarity with its neighbours and also to test its significance. Five scenarios may emerge:

1. Locations with high values with similar neighbours: high-high. Also known as hot spots

2. Locations with low values with similar neighbours: low-low. Also known as cold spots

3. Locations with high values with low-value neighbours: high-low. Potential "spatial outliers.

4. Locations with low values with high-value neighbours: low-high. Potential "spatial outliers.

5. Locations with no significant local autocorrelation.

These specific configurations can be first identified from a scatter plot showing observed values against the averaged value of their neighbours. This so-called Moran scatter plot is a useful exploratory tool. Once a significance level is set, values can also be plotted on a map to display the specific locations of hot spots and potential outliers.

Recently, LISA has been successfully used in various research areas outside geography: to identify and test "hot spots" (positive autocorrelation or similarity) and "cold spots" (negative autocorrelation or dissimilarity) in biological data sets (Sokal et al., 1998a, 1998b), to characterize remote sensing and Landsat TM imagery (Wulder and Boots, 1998 and 2001), to detect spatial clustering of disease (i.e., spatial scan statistic) in epidemiological studies (Hjalmars et al., 1996; Kulldorff, 1997; Gangnon and Clayton, 2001), and to study the spatial point patterns in a minefield and in a plot of pine saplings (Cressie and Collins 2001a and 2001b). LISA is commonly formulated as a numerical measure of a random variable relating each individual datum to the values observed at neighboring locations. It is similar or compatible, at least conceptually, to traditional tree competition indices. Therefore, LISA would be an appropriate indicator for local spatial heterogeneity and useful for investigating the effects of neighboring trees of different species, sizes, and separation distances on the subject tree's performance. Furthermore, LISA can be readily incorporated into visualization tools, such as a geographic information system (GIS), because it provides geo-referenced information at a local level (Unwin, 1996 and Lee and Wong, 2001).

Shi and Zhang (2003) have utilized various LISA approaches to explore the relationship between LISA and traditional tree competition indices, to explore the possibility that LISA can be used as a predictor variable for predicting tree growth, and to demonstrate the usefulness of LISA for identifying and testing clusters of trees with similar or dissimilar sizes. They shows that like most of the competition indices, LISA had moderate correlations with tree basal area growth. For predicting the tree basal area growth in a linear regression model, the local Gi performed better than many $(73 \%)$ competition indices at a plot aggregation level and had higher explanatory power than most $(91 \%)$ competition indices at an individual plot level. Therefore, they recommended that LISA can replace the traditional competition indices for exploring the competitive status of neighboring trees, investigating the relationships between tree competition and growth, and estimating individual tree growth as a predictor variable in a forest growth simulator. The hot spots or cold spots identified by LISA provide useful information for the design of silvi-cultural and management treatments, such as selection thinning.

The second requirement of the LISA permits the decomposition of a global coefficient of spatial autocorrelation into separate parts, making it possible to identify the individual locations that are major contributors to the global autocorrelation. Anselin (1995) described two LISAs to match the established global SA coefficients, $I$ and $c$. These LISAs employ only those elements $w_{i j}$ of the weight matrix $W$ that have nonzero weights with locality $i$, whose local spatial 
Table 1. Statistical relationships between $\mathrm{z}$ and $\mathrm{p}$ values (Mathur 2014).

\begin{tabular}{lcc}
\hline z-score (Standard deviations) & p-value (Probability) & Confidence level \\
\hline$<-1.65$ or $>+1.65$ & $<0.10$ & $90 \%$ \\
$<-1.96$ or $>+1.96$ & $<0.05$ & $95 \%$ \\
$<-2.58$ or $>+2.58$ & $<0.01$ & $99 \%$ \\
\hline
\end{tabular}

Table 2. Interpretation of the $\mathrm{z}$ and $\mathrm{p}$ values (Mathur 2014).

The p-value is not statistically significant. We cannot reject the null hypothesis. It is quite possible that the spatial distribution of feature values is the result of random spatial processes. The observed spatial pattern of feature values could very well be one of many, many possible versions of complete spatial randomness (CSR).

The p-value is statistically significant, and We may reject the null hypothesis. The spatial distribution of high the $\mathrm{z}$-score is positive. values and/or low values in the dataset is more spatially clustered than would be expected if underlying spatial processes were random.

The p-value is statistically significant, and We may reject the null hypothesis. The spatial distribution of high the $\mathrm{z}$-score is negative. values and low values in the dataset is more spatially dispersed than would be expected if underlying spatial processes were random. A dispersed spatial pattern often reflects some type of competitive process - a feature with a high value repels other features with high values; similarly, a feature with a low value repels other features with low values.

autocorrelation we wish to evaluate. The local Moran's coefficient at locality is defined as

$$
M C_{i}=\left(X_{i}-\mathrm{X}\right) \sum_{j} c_{i j}(d)\left(X_{j}-\mathrm{X}\right)
$$

A positive $M C_{i}$ indicates a spatial clustering of similar values (either high or low), whereas a negative $M C_{i}$ indicates a clustering of dissimilar values (e.g., a location with low values is surrounded by neighbors with high values). When the $M C_{i}$ is standardized by division by the variance.

$$
\left.\frac{\sum\left(X_{j}-\dot{x}\right)^{2}}{n}\right)
$$

A pseudo-significant level of $M C_{i}$ can be obtained by a conditional randomization or permutation approach. A small $P$ value (e.g., $P<0.05$ ) indicates that location $i$ is significantly associated with the relatively high values of the surrounding locations. A large $P$-value (e.g., $\mathrm{P}>$ 0.95 ), on the other hand, indicates that location $i$ is significantly associated with relatively low values of the surrounding locations. Anselin (1995) outlined a local variant of the Geary ratio

$$
G R_{i}=\sum_{j} c_{i j}(d)\left(X_{i}-\dot{X}_{j}\right)^{2}
$$

Unlike the local Moran, the local Geary is a measure of the weighted sum of square differences between the observed values at location $i$ and those of its surrounding locations. The calculation of the pseudo-significant level $P$-value is similar to that of the local Moran after the standardization. A small $P$-value $(P<0.05)$ indicates a large $G R_{i}$ in extremes, which suggests a positive spatial association (i.e., similarity) of observation $i$ with its surrounding observations, whereas a large $P$-value $(P$ $>0.95$ ) indicates a small $G R_{i}$ in extremes, which suggests a negative spatial association (i.e., dissimilarity) of observation $i$ with its surrounding observations. From their previous work (Getis and Ord 1992), Ord and Getis (1995) defined local G statistics, which give an index of spatial clustering of a set of observations over a defined neighborhood

$$
G_{i}^{*}=\frac{\sum_{j} c_{i j}(d) X_{j}}{\sum_{j} X_{j}} i=j
$$

The two equations differ depending on whether the location $i$ around the clustering is measured is included in the calculation. There is no theory to guide which one to use in any particular situation, but the difference between the two is typically small. The $G i$ or $G i *$ compares the local (weighted) average with the global average. If high values of $X$ (i.e., the variable under study) tend to cluster around $i$, the $G_{i}$ or $G_{i}$ * will be high; if low values of $X$ tend to cluster around $i$, then the $G i$ or $G i$ * will be low; and no distinct clustering of high or low values of $X$ around $i$ will produce intermediate values of the $G_{i}$ or $G_{i}$ * (Fotheringham et al., 2000). After the standardization, a significant and positive $G_{i}$ or $G_{i} *$ indicates that the location $i$ is surrounded by relatively large values, whereas a significant and negative 
$G i$ or $G i *$ indicates that the location $i$ is surrounded by relatively small values. The $G_{i}$ or $G_{i} *$ statistics can be used to identify spatial agglomerative patterns with high-value clusters or low-value clusters. In summary, the local $M C_{i}$ measures joint covariance of neighboring locations. In other words, it measures the correlation of each $X i$ with all $X_{j s}$ within the distance $d$ of the subject location $i$. If these locations deviate significantly from the average in a given area and have similarly high or low values with the subject point, the local spatial autocorrelation is positive. If the subject location is widely different from the mean and has values unlike those of its neighbors, the local spatial autocorrelation is negative. The local $G R_{i}$ measures (squared) differences of the values between the subject and the neighboring locations. High values of $G R_{i}$ indicate negative spatial autocorrelation. Data values at the subject location and its neighbors that are all close to the mean are shown as a positive spatial autocorrelation by the $G R_{i}$, but as weak or 0 autocorrelation by the $M C_{i}$. The local $G_{i}$ and $G_{i} *$ measure overall concentration or lack of concentration of all pairs of $(X i, X j)$ such that $i$ and $j$ are within the distance of each other. The $G_{i}$ and $G_{i} *$ tend to agree more with the $M C_{i}$ than with the $G R_{i}$ (Getis and Ord 1996, Anselin 1995, Sokal et al., 1998a and 1998b).

Variogram approach: Geo-statistical methods deal with the question of how variance and covariance depend on the instance between observations (i.e., quadrats). Spatial autocorrelation, or distance dependence, is commonly modelled by fitting a variogram function to an empirical variogram (Isaaks and Srivastava, 1989; Haining, 1990; Cressie, 1991; Burrough and McDonnell, 1998). An empirical variogram is a plot of half the squared difference between two observations (the semi-variance) against their distance in space, averaged for a series of distance classes. A simple variogram model is defined by the model family and the parameters sill (the average half squared difference of two independent observations), range (the maximum distance at which pairs of observations will influence each other), and nugget (the variance within the sampling unit).

A correlogram is a graph in which autocorrelation values are plotted, on the ordinate, against distance classes among sites on the abscissa. Correlograms provide evidence for the autocorrelation intensity, the size of the zone of influence and the type of spatial pattern of the variable under study. The shape of a correlogram gives indications about the spatial pattern of the variable, as well as about the underlying generating process (Sokal 1986; Legendre and Fortin, 1989). Inference about the underlying generating process can be made from the shape of the correlogram only when the correlogram is globally significant; Oden (1984) and Legendre and Fortin (1989) show how to compute such a global test, whose aim is to correct for simultaneous multiple testing.

Correlograms (Cliff and Ord 1981) can be computed for single variables (Moran's I or Geary's $c$ autocorrelation coefficients) or for multivariate data (Mantel correlogram). In all cases, a test of significance is available for each individual autocorrelation coefficient plotted in a correlogram. Similarly, a variogram is a graph in which semi-variance is plotted, on the ordinate, against distance classes among sites on the abscissa. In the geo -statistical tradition, semi-variance statistics are not tested for significance, although they could be through the test developed for Geary's $C$, when the condition of second order stationary is satisfied. Statistical models may be fitted to variograms (linear, exponential, spherical, Gaussian, etc.); they allow the investigator to relate the observed structure to hypothesized generating processes or to produce interpolated maps by kriging. Because they measure the relationship between pairs of observation points located a certain distance apart, correlograms and variograms may be computed either for preferred geographic directions or, when the phenomenon is assumed to be isotropic in space, in an all-directional way. Huo et al. (2012) have plotted spatial correlogram with Moran's I. They have used this tool for mapping of soil heavy metals in Beijing, China. According to Legendre and Fortin (1989), the spatial correlogram can be standardized into a standardized correlogram, in which the ordinate is standardized Moran's I and Z. The shape of the standardized correlogram provides inductions about the spatial pattern (spatial clusters and spatial outliers) and spatial correlation distance of a variable (Zhang et al., 1995). However, the standardized correlogram often has one or more positive correlation ranges. Zhang et al. (1998) have explained that the closer positive correlation range represents the average size of the zone of spatial clusters, that is, the spatial correlation distance.

Semi-Variogram: Calculating semi variogram (called variograms for simplicity) is a basic geo-statistical measure to determine the rate of change of a regionalized variable along a specific orientation (usually distances). Semivariogram values are defined as the sum of squared differences between pairs of points separated by a certain distance divided by two times the number of points in a distance class. By plotting experimental semi-variogram values against distance classes in a graph, es obtain a semi-variogram. Like correlograms, semi variograms (decompose the spatial (or temporal) variability of observed variables among distance classes. The structure function plotted as the ordinate, called semi-variance, is the numerator of equation given for Geary's $\mathrm{C}$ function.

$$
\gamma(d)=\frac{1}{2 W} \sum_{n=1}^{n-1} \sum_{i=h+1}^{n} W_{h i}\left(y_{n}-y_{i}\right)^{2}
$$

$\gamma(d)$ is thus a non-standardized form of Geary's $c$ coefficient. $\gamma$ may be seen as a measure of the error mean square of the estimate of $y i$ using a value $y h$ distant from it by $d$. The calculation is repeated for different values of $d$. This provides the sample variogram, which is a plot of 

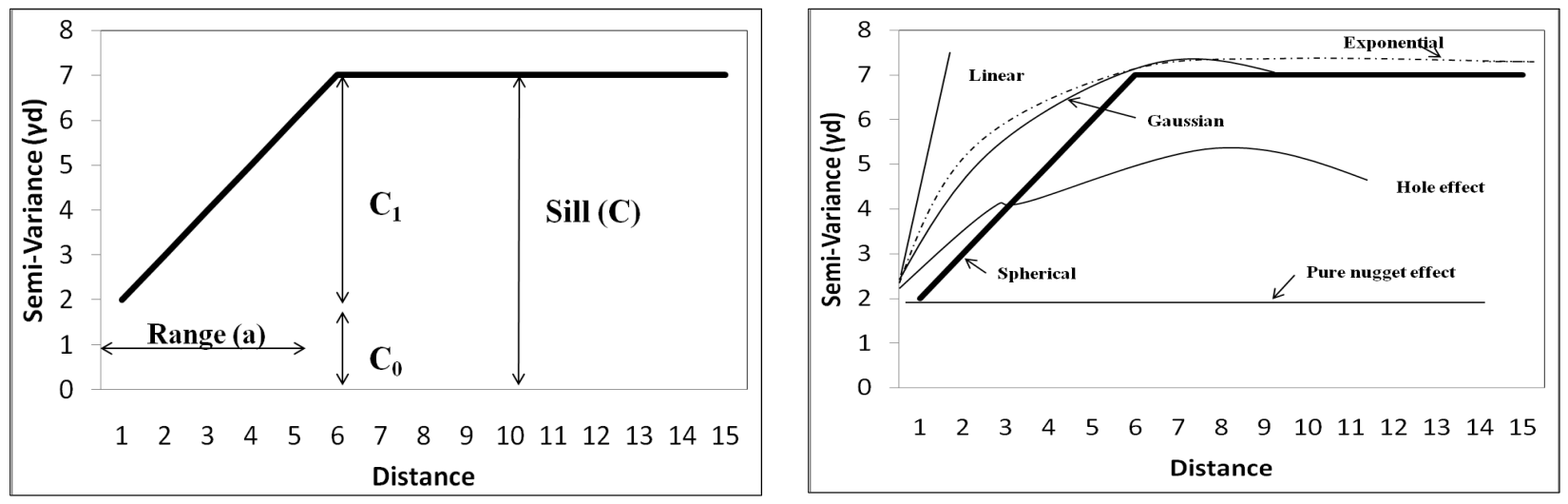

Fig. 2. Types of semi-variance plots (Robertson 2008).

the empirical values of variance $\mathrm{g}(d)$ as a function of distance $d$.

Generally, variograms tend to level off at a sill which is equal to the variance of the variable the presence of a sill implies that the data are second-order stationary. The distance at which the variance levels off is referred to as the range (parameter $a$ ); beyond that distance, the sampling units are not spatially correlated. The discontinuity at the origin (non-zero intercept) is called the nugget effect; the geo-statistical origin of the method transpires in that name. It corresponds to the local variation occurring at scales finer than the sampling interval, such as sampling error, fine-scale spatial variability, and measurement error. The nugget effect is represented by the error term $* i j$ in spatial structure model. It describes a portion of variation which is not autocorrelated, or is autocorrelated at a scale finer than can be detected by the sampling design. The parameter for the nugget effect is $C_{0}$ and the spatially structured component is represented by $C_{1}$; the sill, $C$, is equal to $C_{0}+C_{1}$. The relative nugget effect is $C_{0} /\left(C_{0}+C_{1}\right)$.

Commonly used variogram models: Although a sample variogram is a good descriptive summary of the spatial contiguity of a variable, it does not provide all the semi -variance values needed for kriging. A model must be fitted to the sample variogram; the model will provide values of semi-variance for all the intermediate distances. According to Robertson (2008) there are four major types of model extensively utilized for variogram approach and mathematical expression of these models are as follows:

Spherical Model $\gamma(d)=C_{0}+C_{1}\left[1.5 \frac{d}{a}-0.5\left(\frac{d}{a}\right)^{3}\right]$ if $d \leq a, \gamma(d)=[$ if $d>a$

Exponential Madel: $\gamma(d)=C_{0}+C_{1}\left[1-\exp \left(-3 \frac{d}{a}\right)\right]$

Gaussian Modeli $\gamma(d)=C_{0}+C_{1}\left[1-\exp \left(-3 \frac{d^{2}}{a^{2}}\right)\right]$
Hole effect Model: $\gamma(d)=C_{0}+C_{1}\left|1-\frac{\sin (a d)}{a d}\right|$. An equivalent form is

where $a^{\prime}=\frac{1}{a} \cdot\left(C_{0}+C_{1}\right)$

Represents the value of $\mathrm{g}$ towards which the dampening sine function tends to stabilize. Linear model: $\gamma(\mathrm{d})=C_{o}+b d$ where $\mathrm{b}$ is the slope of the variogram model. A linear model with sill is obtained by adding the specification: $\gamma(\mathrm{d})=\mathrm{C}$ if $\mathrm{d}^{3}$ a. Pure nugget effect model: $\gamma(\mathrm{d})=C_{o}$ if $\mathrm{d}>\mathrm{o} ; \gamma(\mathrm{d})=$ $O$ if $\mathrm{d}=0$. The second part applies to a point estimate. In practice, observations have the size of the sampling grain the error at that scale is always larger than 0 .

\section{Conclusion}

Spatial autocorrelation is a widespread phenomenon in ecology. The spread of plants by the processes of growth, vegetative reproduction, and limited seed dispersal produces spatial autocorrelation etc are associated with spatial autocorrelation of plants. Spatial autocorrelation can be analyzed from two different perspectives. Global autocorrelation analysis involves the study of the entire map pattern and generally asks the question as to whether the pattern displays clustering or not. Local autocorrelation, on the other hand, shifts the focus to explore within the global pattern to identify clusters or so called hot spots that may be either driving the overall clustering pattern, or that reflect heterogeneities that depart from global pattern. Both types of methods have been extensively utilize in biological data sets, to characterize remote sensing and Landsat TM imagery, to detect spatial clustering of disease in epidemiological studies etc. The study revealed the dominance of Moran's I approach for their extensive use compared to other methods. Our review suggested the lesser efforts of using this technique for plant population in the Indian subcontinent; therefore there is an ample scope to utilize these techniques to quantify the spatial relationships between different plants variables located on different habitats. 


\section{REFERENCES}

Almeida-Neto, M. and Lewinsohn, T.M. (2004). Small -scale spatial autocorrelation and the interpretation of relationships between phenological parameters. Journal of Vegetation Sciences, 15(4): 561-568.

Anselin, L. (1995). Local Indicators of Spatial Association -LISA. Geographical Analysis

Anselin, L. (2002). Under the hood: issues in the specification and interpretation of spatial regression models. - Agriculture Economics, 17: 247-267.

Assuncao, R.M. and Reis, E.A. (1999). A new proposal to adjust Moran's I for population density. Statistics in Medicine, 18(16): 2147-2162.

Besag, J. (1974). Spatial interaction and the statistical analysis of lattice system. Journal of the Royal Statistitical Society, 36: 192-225.

Boots, B.N. and Getis, A. (1988). Point Pattern Analysis, Newburry Park, CA, Sage Publication

Burrough, P.A. and McDonnell, R.A. (1998). Principles of Geographical Information Systems. Spatial Information Systems and Geostatistics. Oxford University Press, New York, 333

Chen, X., Li, B.L. and Zhang, X.S. (2008). Using spatial analysis to monitor tree diversity at large scale: a case study in Northeast China transect. Journal of Plant Ecology, 1(2): 137-141.

Chou, Y.H. (1997). Exploring spatial analysis in geographic information systems. Santa Fe: Onward Press.

Chuang, K.S. and Huang, H.K. (1992). Assessment of noise in a digital image using the joint-count statistic and the Moran test. Physics in Medicine and Biology, 37 (2): 357-369.

Cliff, A.D. and Ord, J.K. (1981). Spatial Processes, Models and Applications. London, Pion, pp. 34-41.

Congalton, R.G. (1988). Using spatial autocorrelation analysis to explore the errors in maps generated from remotely sensed data. Photogrammetric Engineering and Remote Sensing, 54: 587-592.

Cressie, N. (1991). Statistics for Spatial Data. Wiley, New York.

Cressie, N. and Collins L.B. (2001a). Patterns in spatial point locations: Local indicators of spatial association in a minefield with clutter. Naval Research Logistic, 48:333-347.

Cressie, N. and Collins, L.B. (2001b). Analysis of spatial point patterns using boundless of product density LISA functions. Journal of Agricultural Biology and Environment, 6:118-135.

Dale, M.T. (1999). Spatial pattern analysis in plant ecology. Cambridge University Press, Campridge, UK.

Diniz-Filho, J.A.F., Bini, L.M. and Hawkins, B.A. (2003). Spatial autocorrelation and red herrings in geographical ecology. Global Ecology and Biogeography, 12: 53-64.

Dormann, C. F. (2007). Effects of incorporating spatial autocorrelation into the analysis of species distribution data. Global Ecology and Biogeography, 16: 129-138.

Dormann, C.F., Jana, M.M., Miguel, B.A., Roger, B., Bolliger, J., Carl, G., Davies, R.G., Hirzel, A., Jets, W., Kissling, O., Kuhn, I., Ohlemuller, R., Neto-Peres, P.R., Reineking, B., Schroder, B., Schurr, F.M. and Wilson, R. (2007). Methods to account for spatial autocorrelation in the analysis of species distributional data: a review. Ecography, 30: 609-628.

Dray, S., Said, S. and Debias, F. (2008). Spatial ordination of vegetation data using a generalization of wartenberg's multivariate spatial correlation. Journal of Vegetation Science, 19 (1): 45-56.

Fortin, M.J. and Dale, M.R.T. (2005). Spatial Analysis - A Guide for Ecologists. Cambridge University Press.

Fortin, M.J., Drapeau, P. and P. Legendre. (1989). Spatial autocorrelation and sampling design in plant ecology. Vegetatio, 83:209-222.

Fotheringham, A.S., Burnsdon, S. and Charlton, M. (2000). Quantitative geography: Perspectives on spatial data analysis. Sage Publications, Thousand Oaks, CA. pp 270.

Fox, E., Balram, S., Dragicevis, S. and Roberts, A. (2012). Spatial analysis of high resolution aerial photographs to analyse the spread of mountain pine beetle infestation. Journal of Sustainable development, 5 (9): 106-129.

Gangnon, R.E. and Clayton, M.K. (2001). A weighted average likelihood ratio test for spatial clustering of disease. Statistics in Medicine, 20:2977-2987.

Geary, R. (1954). The contiguity ratio and statistical mapping. The Incorporated Statistician 5: pp115-45

Getis, A. (1991). Spatial interaction and spatial autocorrelation: across-product approach Environment and Planning A, 23: $1269-1277$.

Getis, A., and Ord, J. K. (1992). The analysis of spatial association by use of distance statistics. Geographical Analysis, 24 (3): $189-206$

Getis, A. and Ord, J.K. (1996). Local spatial statistics: An overview. Spatial analysis: Modeling in a GIS environment. Longley, P., and M. Batty (eds.). Wiley, New York. pp. 261-277.

Gibson, D.J. (2002). Methods in Comparative Plant Population Ecology, Oxford University, UK. 344.

Goodchild, M. F. (1986). Spatial Autocorrelation. Catmog 47, Geo Books.

Goslee, S.C. (2006). Behavior of vegetation sampling methods in the presence of spatial autocorrelation. Plant Ecology, 187: 203-2012.

Griffith, D.A. (2003). Spatial autocorrelation and spatial filtering: gaining understanding through theory and scientific visualization. Berlin, Germany: Springer-Verlag.

Griffith, D.A. and Peres-Neto, P.R. (2006). Spatial modeling in ecology: the flexibility of eigen function spatial analyses in exploiting relative location information. Ecology, 87: 2603-2613.

Haining, R. (1990). Spatial Data Analysis in Social and Environmental Sciences. Cambridge University Press: New York.

Hawkins, B.A., Field, R., Cornell, H.V., Currie, D.J., Guegan, J.F., Kaufman, D.M., Kerr, J.T., Mittelbach, G.G., Oberdorff, T., O’Brien, E.M., Porter, E.E. and Turner, J.R.G. (2003). Energy, water, and broad-scale 
geographic patterns of species richness. Ecology, 84: 3105-3117

Heikkinen, R.K. (1996). Predicting patterns of vascular plant species richness with composite variables: a meso-scale study in Finnish Lapland. Vegetatio, 126: 151-165.

Henebry, G.M. (1995). Spatial model error analysis using autocorrelation indices. Ecological Modelling 82: 75-91.

Hjalmars, U., Kulldorff, M., Gustafsson G. and Nagarwalla, N. (1996). Childhood leukemia in Sweden: Using GIS and a spatial scan statistic for cluster detection. Statistics in Medicine, 15:707-715.

Hubert, L., Golledge, R. and Costanzo, C.M. (1981). Generalized procedures for evaluating spatial autocorrelation. Grographical Analysis, 13: 224-233

Huo, X., N., Li, H., Sun, D.F., Zhou, L.D. and Li, B.G. (2012). Combining geostatistics with Moran's I analysis for mappings soil heavy metals in Beijing, China. International Journal of Environmental Research and Public Health, 9: 995-1017.

Isaaks, E.H. and Shrivastava, R.M. (1989). An introduction to applied geo-statistics. - Oxford University Press.

Jackson, M.C. and Waller, L.A. (2005). Exploring Goodness -of-fit and spatial correlation using components of Tango's Index of spatial clustering. Geographical Analysis, 37(4):371-382.

Jackson, M.C., Huang, L., Xie, Q and Tiwari, R. (2010). A modified version of Moran's I. International Journal of Health Geographic, 9: 33-43.

Koenig, W.D. (1998). Spatial autocorrelation in California land birds. Conservation Biology, 12:612-620.

Kühn, I. (2007). Incorporating spatial autocorrelation may invert observed patterns. Diversity and Distribution, 13 (1): 66-69.

Kulldorff, M. (1997). A spatial scan statistic. Community Statics Theory and Methods, 26:1481-1496.

Laffan, S.W. (2006). Assessing regional scale weed distributions, with an Australian example using Nassella trichotoma. Weed Research, 46(3): 194-206.

Lee, J. and Wong, D.W.S. (2001). Statistical analysis with ArcView GIS. Wiley, New York. 192 p.

Legendre, L. and Legendre, P. (1984). 1 cologie numrrique. 2irme ed. Tome 2: La structure des donnres 6cologiques. Masson, Paris et les Presses de l'Universit6 du Quebec.

Legendre, P. (1993). Spatial autocorrelation: trouble or new paradigm? Ecology, 74: 1659-1673.

Legendre, P. and Fortin, M.J. (1989). Spatial pattern and ecological analysis. Vegetatio, 80: 107-38.

Legendre, P. and Legendre, L. (1998). Numerical Ecology. Elsevier. USA.

Legendre, P., Dale, M.T.R., Fortin, M.J., Gurvitch, J., Hohn, M. and Myers, D. (2002). The consequences of spatial structure for the design and analysis of ecological field surveys. - Ecography, 25: 601-615.

Lennon, J.J. (2000). Red-shifts and red herrings in geographical ecology. Ecography, 23: 101-113.

Mathur, M. (2014). Attributes of Plant Spatial Analysis. Today and Tomorrow Printer and Publishers, New Delhi, India.

Miller, J. Franklin, J. and Aspinall, R. (2007). Incorporating spatial dependence in predictive vegetation models. Ecological Modeling, 202: 225-242.

Moran, P.A.P. (1948). The interpretation of statistical maps. Journal of the Royal Statistical Society, Series B, 10: 243-251.

Mueller-Warrant, G.W., Whittaker, G.W. and Young, W.C.
(2008). GIS analysis of spatial clustering and temporal change in weeds of grass seed crops. Weed Science, 56 (5): 647-669.

Oden N. (1995). Adjusting Moran's I for population density. Statatistical Medicine, 14(1):17-26.

Oden N.L. (1984). Assessing the significance of a spatial correlogram. Geographical Analysis, 16: 1-16.

Odland, J. (1988). Spatial autocorrelation. In: G.I. Thrall (Ed.), Sage University Scientific Geography Series no. 9. Sage Publications, Beverly Hills. pp 87.

Ord, J.K and Getis, A. (1995). Local spatial autocorrelation statistics: distributional issues and an application. Geographical Analysis, 27: 286-306.

Overmars, K.P., Koning, G.H.I. and Velkamp, A. (2003). Spatial autocorrelation in multi-scale land use models. Ecological Modeling, 164: 257-270.

Palma, L. Beja, P. and Rodrigues, M. (1999). The use of sighting data to analyze Iberian lynx habitat and distribution. Journal of Applied Ecology, 36: 812- 824.

Perry, J.N., Liebhod, A.M., Rosenbery, M.S., Dungan, I., Miriti, M., Jakomulska, A. and Citron-Pousty, S (2002). Illustrations and guidelines for selecting statistical methods for quantifying spatial paterrns in ecological data. Ecography, 25: 578-600.

Pugh, S. and Congalton, R. (2001). Applying spatial autocorrelation analysis to evaluate error in New England forest cover type maps derived from Landsat Thematic Mapper Data. Photogrammetric Engineering and Remote Sensing, 67 (5): 613-620.

Radeloff, V.C., Miller, T.F., He, H. S. and D..J. Mladenoff. (2000). Periodicity in landscape pattern and geostatistical models: autocorrelation between patches. Ecography, 23: 81-91.

Robertson G.P. (2008). GS ${ }^{+}$Geostatistics for the environmental sciences. Gamma Design Software, Plainwell, Michigan USA. P. 165.

Roe, C.M., Parker, G.C., Korsten, C.A., Lister, C.J., Weatherall, S.B., Lawrence Lodge, R.H, E. and Wilson, B.J. (2012). Small-scale spatial autocorrelation in plant communities: the effects of spatial grain and measure of abundance, with an improved sampling scheme. Journal of Vegetation Science, 23: 471-482.

Rogerson P. (1999). The detection of clusters using a spatial version of the Chi- Square Goodness-of-Fit Statistic. Geographical Analysis, 31(1):128-147.

Shi, H., and Zhang, L. (2003). Local analysis of tree competition and growth. Forest Science, 49 (6): 938-946.

Sokal, R.R. and Oden, N.L. (1978). Spatial autocorrelation in biology. 1. Methodology. Biological Journal of Linnaeus Society, 10: 199-228.

Sokal, R.R. (1986). Spatial S data analysis and historical processes. In: Diday, E. (eds), Data analysis and informatics, IV. Proceedings of the Fourth International Symposium on Data Analysis and Informatics, pp. 29-43. Versailles, France, North-Holland, Amsterdam.

Sokal, R.R., Oden, N.L. and Thomson, B.A. (1998a). Local spatial autocorrelation in a biological model. Geographical Analysis, 30:331-354.

Sokal, R.R. Oden, N.L. and Thomson, B.A. (1998b). Local spatial autocorrelation in a biological variables. Biological Journal of Linnaeus Society, 65:41-62.

Su-Wei, F. and Hsieh, C.F. (2010) spatial autocorrelation patterns of understory plant species in a subtropical rainforest at Lanjenchi, Southern Taiwan. Taiwan, 55: 160-171. 
Suzuki, S.N., Kachi, N. and Suzuki, J.I. (2008). Development of a local size hierarchy causes regular spacing of trees in an even-aged Abies forest: Analyses using spatial autocorrelation and the mark correlation function. Annals of Botany, 102(3): 435-441.

Tobin, P.C. (2004). Estimation of the spatial autocorrelation function: consequences of sampling dynamic populations in space and time. Ecography, 27(6): 767-775.

Torgersen, C.E., Jones, J.A., Moldenke, A.R. and LeMaster, M.P. (1995). The spatial heterogeneity of soil invertebrates and edaphic properties in an old growth forest stand in western Oregon. Pages 225-236 in H.P. Collins, G.P. Robertson, and M.J. Klug, editors. The significance and regulation of soil biodiversity. Kluwer Academic Publishers, Dordrecht, Netherlands.

Unwin, D.J. (1996). GIS, spatial analysis and spatial statistics. Progress in Human Geography. 20:540-551.

Upton, G.J. and Fingleton, B. (1985). Spatial data analysis by example, volume 1: Point pattern and quantitative data. Wiley, Toronto Singapore, Brisbane, New York, Chichester

Waldhor T. (1996). The spatial autocorrelation coefficient Moran's I under heteroscedasticity. Statistics in Medicine, 15(7-9):887-892.

Waller, L.A. and Gotway, C.A. (2004). Applied Statistics for Public Health Data. New York: Wiley.

Waller, L.A., Hill, E.G. and Rudd, R.A. (2006). The geography of power: statistical performance of tests of clusters and clustering in heterogeneous populations. Statistics in Medicine, 25(5):853-865.

Weixelman, D.A. and Riegel, G.M. (2012). Measurement of spatial autocorrelation of vegetation in mountain meadows of the Sierra-Nevada, California and Western Nevada. Madrono, 59: 143-149.

Wulder, M. and Boots, B. (1998). Local spatial autocorrelation characteristics of remotely sensed imagery assessed with the Getis statistic. International Journal of Remote Sensing, 19: 2223-2231.

Wulder, M. and Boots B. (2001). Local spatial autocorrelation characteristics of Landsat TM imagery of a managed forest area. Canadian Journal of Remote Sensing, 27:67 -75 .

Zhang, C.S. and McGrath, D. (2004). Geostatistical and GIS analyses on soil organic carbon concentrations in grassland of south-eastern Ireland from two different periods. Geoderma, 119: 261-275.

Zhang, C.S., Tao, S., Yuan, G.P. and Liu, S. (1995). Spatial autocorrelation analysis of trace element contents of soil in Tianjin plain area (in Chinese, with English abstract). Acta Pedology Sinica, 32: 50-57.

Zhang, C.S. Zhang, S. and He, J.B. (1998). Spatial distribution characteristics of heavy metals in the sediments of Changjiang River system-Spatial autocorrelation and fractal methods (in Chinese, with English abstract). Acta Geogaphyr Sinica, 53: 87-96. 